\title{
Effects of cytochrome P450 family 3 subfamily A member 5 gene polymorphisms on daunorubicin metabolism and adverse reactions in patients with acute leukemia
}

\author{
ZHEN HUANG $^{1}$, JUXIANG WANG ${ }^{1}$, JIANGCHAO QIAN ${ }^{1}$, \\ YUAN LI ${ }^{1}$, ZHISHENG XU ${ }^{1}$, MIN CHEN ${ }^{1}$ and HONGFEI TONG ${ }^{2}$ \\ Departments of ${ }^{1}$ Hematology and ${ }^{2}$ Hepatobiliary Surgery, The Second Affiliated Hospital and \\ Yuying Children's Hospital of Wenzhou Medical University, Wenzhou, Zhejiang 325027, P.R. China
}

Received January 27, 2016; Accepted January 20, 2017

DOI: $10.3892 / \mathrm{mmr} .2017 .6470$

\begin{abstract}
The present study aimed to investigate the association between the genetic polymorphism of cytochrome P450 family 3 subfamily A member 5 (CYP3A5) and the activity of CYP3A and plasma concentrations of daunorubicin (DNR) in patients with acute leukemia. A total of 36 children with newly diagnosed acute lymphoblastic leukemia were enrolled in the study. Polymerase chain reaction (PCR)-restriction fragment length polymorphism and PCR product sequencing were used to detect the genotype of CYP3A5*3. PCR was then used to express the mRNA expression of CYP3A5. A midazolam probe method was used to detect CYP3A enzyme activity, and DNR concentrations were measured using high performance liquid chromatography. Children with different genotypes had different mRNA expression levels of CYP3A5, and CYP3A enzyme activity in children with the CYP3A $5 * 1$ allele was higher, compared with that in children with the CYP3A $5 * 3$ allele. In addition, the area under the curve (AUC) $)_{0-24 \mathrm{~h}}$ and $\mathrm{AUC}_{0-\infty}$ of DNR were significantly different in children with different genotypes, however, no statistically significant differences were found in half-life or maximum concentration. The AUC of DNR was increased in children with acute lymphatic leukemia who suffered from cardiotoxicity, compared with those in the normal group. The CYP3A5*3 gene polymorphism was closely associated with the mRNA expression of CYP3A5, CYP3A enzyme activity and DNR plasma drug concentration, and exhibited different drug adverse reactions.
\end{abstract}

Correspondence to: Dr Hongfei Tong, Department of Hepatobiliary Surgery, The Second Affiliated Hospital and Yuying Children's Hospital of Wenzhou Medical University, 109 Xueyuan Xi Road, Wenzhou, Zhejiang 325027, P.R. China E-mail: hongfeitong@126.com

Key words: individualized therapy, cytochrome $\mathrm{P} 450$ family 3 subfamily A member 5, gene polymorphism, cytochrome P450 family 3 subfamily A enzyme activity, daunorubicin

\section{Introduction}

Individualized chemotherapy is an increasingly popular type of tumor treatment. Daunorubicin (DNR) is a chemotherapeutic of the anthracycline family, which is used as a treatment for acute leukemia (1). However, this drug has a higher affinity to myocardial tissue than other tissues, which limits the application of DNR due to accumulated cardiotoxicity (2-4). Several studies have confirmed that cytochrome P450 family 3 subfamily A member 5 (CYP3A5) is involved in the clearance of DNR metallization (5). Studies of CYP3A5 have predominantly focused on osteosarcoma, breast cancer and the use of immunosuppressants following liver or kidney transplantation (6-9), however, few studies have reported on the association between CYP3A5 and acute leukemia $(9,10)$. Thus, the present study examined the association between CYP3A5 gene polymorphisms and CYP3A enzyme activity, and plasma concentrations of DNR. The study aimed to provide primary experimental evidence for the use of DNR and reduction of adverse reactions, and improve the individualized treatment of leukemia.

\section{Materials and methods}

Subjects. According to the CCLG-ALL-2008 protocol (11), 36 children with acute lymphatic leukemia (ALL), who were first diagnosed and hospitalized at the Department of Hematology of the Second Affiliated Hospital and Yuying Children's Hospital of Wenzhou Medical University (Wenzhou, China) were enrolled in the present study between January 2009 and July 2013. All patient diagnoses were confirmed by clinical, morphological and immunological examinations, histochemical staining and chromosome karyotype analysis, and included nine cases in the low risk group, 17 cases in the moderate risk group and 10 cases in the high risk group. The ALL treatment regimen comprised vincristine, DNR, L-asparaginase and prednisone administration for 4 weeks. DNR was injected at $30 \mathrm{mg} / \mathrm{m}^{-2}(1 \mathrm{~h})$ for 2-3 days (2 days in low-risk group, 3 days in moderate and high-risk group). Children who did not complete a course of chemotherapy and were discharged from hospital or those who succumbed 
to mortality were excluded from the investigation. The present study was approved by the ethics committee of The Second Affiliated Hospital and Yuying Children's Hospital of Wenzhou Medical University. All family members of the patients provided signed informed consent.

Genotype detection at site 6,986 of CYP3A5. A $300 \mu \mathrm{l}$ sample of bone marrow was collected and stored in tubes with $2 \%$ EDTA. DNA extraction was performed according to the manufacturer's protocol of the Wizard DNA kit (Promega Corporation, Madison, WI, USA). Primer sequences were as follows: Sense 5'-CATCAGTTAGTAGACAGATGA-3' and antisense 5'-GGTCCAAACAGGGAAGAAATA-3'. A polymerase chain reaction (PCR) amplification instrument (ABI 9600; Applied Biosystems; Thermo Fisher Scientific, Inc., Waltham, MA, USA) was used. The reaction volume was as follows: $0.4 \mu \mathrm{mol}-1$ sense primer, $0.4 \mu \mathrm{mol}-1$ antisense primer, 2 ng DNA template, 4 mmol- $\mathrm{MgCl}_{2}, 0.05 \mathrm{U}-\mu 1 \mathrm{Taq}$ DNA polymerase (Promega Corporation), dNTPs (dATP, dCTP, dGTP and dTTP, 0.4 mmol-1 each), and ddH2O was added to bring the final volume to $50 \mu \mathrm{l}$. The PCR conditions were as follows: Denaturation at $94^{\circ} \mathrm{C}$ for $5 \mathrm{~min}$, followed by 35 cycles of $94^{\circ} \mathrm{C}$ for $30 \mathrm{sec}, 55^{\circ} \mathrm{C}$ for $30 \mathrm{sec}$, extension at $72^{\circ} \mathrm{C}$ for $1 \mathrm{~min}$, and final extension at $72^{\circ} \mathrm{C}$ for $7 \mathrm{~min}$. The PCR product $(20 \mu \mathrm{l})$ and restriction enzyme, SspI (New England Biolabs, Ipswich, MA, USA) were incubated at $37^{\circ} \mathrm{C}$ for $4 \mathrm{~h}$, which was followed by $2 \%$ agarose gel electrophoresis, staining with ethidium bromide, observation and capturing of images using a gel image analyzer, respectively. To confirm the results of the gel electrophoresis, the PCR products from seven specimens were directly sequenced to ensure the genotypes of gene variation. The sequencing results were compared with the human genome database of NCBI (https://www.ncbi.nlm. nih.gov/gene/1577), if A became $\mathrm{G}$, it was defined as gene variation.

Detection of gene expression levels of CYP3A5. A 3-4 ml sample of marrow was collected under sterile conditions and stored in tubes with EDTA, followed by immediate single karyocyte extraction, extraction of RNA (TRIzol kit; Invitrogen; Thermo Fisher Scientific, Inc.) and cDNA synthesis (SuperScript Double-Stranded cDNA Synthesis kit; Thermo Fisher Scientific, Inc.) were performed according to the manufacturer's protocols. Primer Express Software v.2.0 was used to design primers according to the CYP3A5 mRNA sequence in GeneBank (https://www.ncbi.nlm.nih.gov/ nuccore/NM_001190484.2). Primers and probes of CYP3A5, wild-type (wt)-CYP3A5, SV1-CYP3A5 and GAPDH (Shanghai Sangon Pharmaceutical Co., Ltd., Shanghai, China) are listed in Table I. The PCR conditions were as follows: Denaturation at $94^{\circ} \mathrm{C}$ for $5 \mathrm{~min}$, followed by 50 cycles of $95^{\circ} \mathrm{C}$ for $15 \mathrm{sec}, 55^{\circ} \mathrm{C}$ for $60 \mathrm{sec}$, and final extension at $72^{\circ} \mathrm{C}$ for $5 \mathrm{~min}$. The PCR product was stored at $4^{\circ} \mathrm{C}$.

Detection of CYP3A activity using amidazolam (MDZ) probe assay. In the morning following a period of nil-by-mouth, the children were injected with $0.1 \mathrm{mg}-\mathrm{kg}$ MDZ solution (Jiangsu Nhwa Pharma Co., Ltd, Jiangsu, China), and $2 \mathrm{ml}$ blood was collected at $0,0.25,0.5,1,2,4$ and $6 \mathrm{~h}$ post-injection, respectively. MDZ standard solutions were prepared at concentrations of 25, 50, 100, 200, 400, 800 and 1,600 ng-ml. Chromatography was performed via Waters 2695 high performance liquid chromatography (HPLC; Waters 2487 UV-VIS detector; Waters Corporation, Milford, MA, USA) on a C18 chromatographic column $(250 \times 4.6 \mathrm{~mm} ; 5 \mu \mathrm{m})$ maintained at a flow rate of $0.6 \mathrm{ml}$-min. The mobile phase consisted of methanol, acetonitrile and potassium phosphate buffer solution (0.02 mol-1; pH7.4) at a ratio of 65:25:10 (v-v), and a $0.45 \mu \mathrm{m}$ filter membrane was used. The wavelength detection was $221 \mathrm{~nm}$ and a quantity of $50 \mu \mathrm{l}$ was added. CYP3A activity was denoted as MDZ clearance (CL) and area under the curve (AUC) (0-t).

HPLC determination of concentrations of DNR and associated metabolized products. A $1 \mathrm{ml}$ sample of blood was collected $15 \mathrm{~min}, 30 \mathrm{~min}$, and 1, 2, 3, 4, 6, 12 and $24 \mathrm{~h}$ following injection, respectively, and stored in tubes with EDTA. The tubes were centrifuged at $4^{\circ} \mathrm{C}$ for $10 \mathrm{~min}$ at $800 \mathrm{x} \mathrm{g}$, and the upper plasma was collected and stored at $-80^{\circ} \mathrm{C}$. An appropriate standard of DNR was weighed and then dissolved in methanol solution (2 mg-ml). Following extraction, blood specimens were added to $200 \mu \mathrm{l}$ mobile phase, followed by mixing for $10 \mathrm{sec}$. The chromatogram conditions were as follows: C18 chromatographic column $(5 \mu \mathrm{m}$; 250x4.6 mm; Fuji Silysia Chemical Ltd., Tokyo, Japan); mobile phase, $0.4 \%$ ammonium acetate solution:acetonitrile at $65: 35$ ratio $(\mathrm{v}-\mathrm{v}) ; \mathrm{pH}=4.0$; temperature, $40.0^{\circ} \mathrm{C}$; flow rate, $1.0 \mathrm{ml}-\mathrm{min}$; volume of sample, $50 \mu \mathrm{l}$. Additionally, the fluorescence detector had a $480 \mathrm{~nm}$ excitation wavelength and $560 \mathrm{~nm}$ emission wavelength. Following appearance, the AUC was calculated. A standard curve was drawn according to the DNR concentration with abscissa and peak area as coordinates. Finally, the DNR concentration was calculated through the peak area and using the standard curve formula ( $Y=481.91, X=985.01 ; R=0.995$; with $Y$ being the peak area and $\mathrm{X}$ the DNR concentration).

Monitoring heart function. According to the standard of acute and subacute toxicity reaction of anticancer drugs (12), echocardiography (ECG), myocardial enzymes and cardiac troponin were monitored at different chemotherapeutic stages in the children with ALL. The DNR accumulated dose and adverse cardiac reactions were observed through follow-up.

Statistical analysis. All statistical analysis was performed using SPSS 19.0. (IBM SPSS, Armonk, NY, USA). One-way analysis of variance was used to compare the difference between two groups. A Games-Howell test was used for multiple comparisons. DAS software (version 2.1.1; Mathematical Pharmacology Professional Committee of China, Shanghai, China) was used to calculate the pharmacokinetic parameters of MDZ and DNR.

\section{Results}

General data. A total of 36 children with ALL were recruited in the present study, including 22 boys and 14 girls with an average age of $7.1 \pm 2.3$ years (range, $3-14$ years old).

Distribution of CYP3A5 genotypes. In the ALL cases, 22 cases were CYP3A5*3-"3, 10 cases were CYP3A5 1 -*3 and four cases 
were CYP3A $5{ }^{*} 1{ }^{*} 1$. Sequence analysis was performed on the three genotypes in the site of 6,986, and the results indicated that the CYP3A5*3-3 genotype had only one peak of G, CYP3A 5*1-1 also had one peak of A, however, two peaks of A and $\mathrm{G}$ were detected in the CYP3A5 $1{ }^{*}{ }^{*} 3$ genotype (Figs. 1-3).

Gene expression of CYP3A5. The CYP3A5"1-"1 genotype had wt-mRNA (wt-CYP3A5), whereas the CYP3A5*1-"3 and CYP3A 5*3-"3 genotypes had two types of mRNA (wt-CYP3A5 and SV1-CYP3A). The wt-mRNA in the different genotypes showed statistical significance (Table II).

Detection of CYP3A enzyme activity and DNR concentration. The CL of MDZ ranged between 0.047 and 0.181, and the AUC ( $0-6$ h) ranged between 390.124 and 1637.427 in the 36 cases. The plasma chromatogram of DNR showed no disturbed peaks prior to or following DNR, and the storage duration was 9.618 min, which suggested that the DNR identification was distinct and that the spots were clear (Fig. 4).

The DNR was gradually reduced in the blood as a two compartment model, and the results of pharmacokinetic parameters were as follows: Half-life $\left(\mathrm{t}_{1-2}\right) \alpha, 0.02-1.25 \mathrm{~h}$; $\mathrm{t}_{1-2} \beta, 3.56-112.72 \mathrm{~h} ; \mathrm{CL}, 14.72-136.69 \mathrm{l} \cdot \mathrm{h}^{-1} \cdot \mathrm{m}^{-2} ; \mathrm{AUC}_{0-24} \mathrm{~h}$, 116.73-832.01 $\mu \mathrm{g} / \mathrm{l}^{-1} \cdot \mathrm{h}$; maximum concentration $\left(\mathrm{C}_{\max }\right)$, 6.85-127.76 $\mu \mathrm{g} / \mathrm{l}^{-1}$, time to maximum concentration $\left(\mathrm{t}_{\max }\right)$, $0.35-1.80 \mathrm{~h}$. These results showed that the DNR parameters were different between individuals; the maximum CL was nine times higher than the minimum, the maximum AUC was seven times higher than the minimum, and the maximum $\mathrm{C}_{\max }$ was four times higher than the minimum.

The children with ALL were divided into three groups, and the pharmacokinetic parameters were analyzed for all cases. The $\mathrm{AUC}_{0-24 \mathrm{~h}}$ and $\mathrm{AUC}_{0-\infty}$ were significantly different among the groups, however, no statistically significant differences were observed in $\mathrm{t}_{1-2} \mathrm{OrC}_{\max }$ (Table III).

Adverse reactions of DNR. Heart ultrasonography and monitoring of cardiac troponin were normal prior to and following chemotherapy in the 36 children. Of these, four children had cardiac dysfunction; three children suffered from an aberrant increase in the myocardial enzyme spectrum, including a lactate dehydrogenase range of 528.1-879.6 U/1, an $\alpha$-hydroxybutyrate dehydrogenase range of 498.2-769.3 U/1 and an aspartate aminotransferase range of 118.2-186.7 U/1. In addition, one child with an abnormal ECG was treated with DNR for 5 months, which was followed by right bundle branch block. A year later, the child succumbed to mortality from acute myocarditis.

\section{Discussion}

In previous years, there has been substantial development in understanding the chemotherapeutics mechanisms of pharmacogenetics and pharmacogenomics (13-15). The cytotoxic effect of chemotherapeutics against tumor cells has significant associations with the expression of specific genes or gene polymorphisms. The detection of associated genes to predict effects in order to select appropriate drugs for individualized chemotherapy has become a rationale for improving effects and reducing the occurrence of ineffective treatment. 


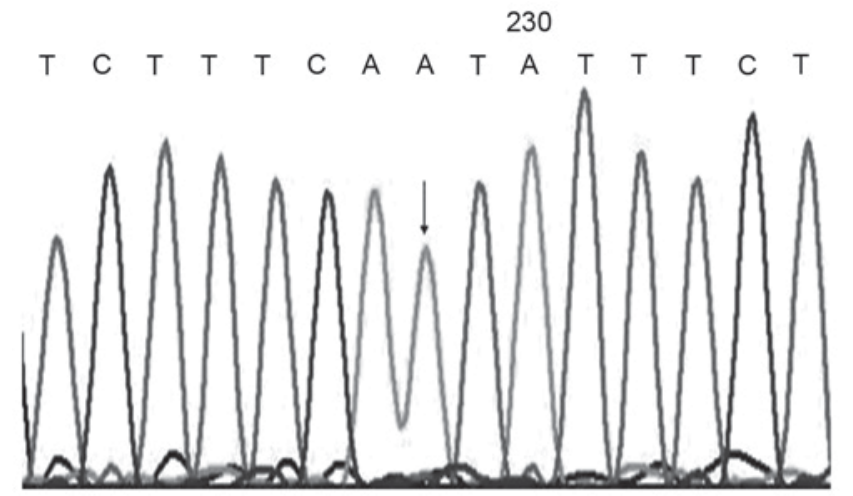

Figure 1. Cytochrome P450 family 3 subfamily A member $5^{*} 1-^{*} 1$ gene sequencing diagram. Arrow shows one peak of A in the site of 6,986.

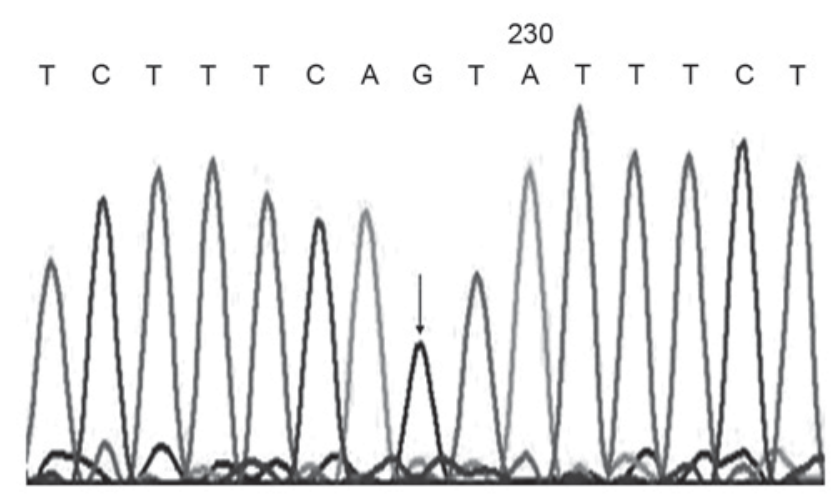

Figure 2. Cytochrome P450 family 3 subfamily A member $5 * 3-3$ gene sequencing diagram. Arrow shows one peak of $\mathrm{G}$ in the site of 6,986 .

DNR is an anthracycline anticancer drug used to treat common acute leukemia. The effect and toxicity of DNR is correlated with blood-concentrations and duration of action, however, there are certain differences among individuals. Studies have confirmed that the CYP3A5 enzyme is involved in the metabolism and clearance of DNR, however, the activity of CYP3A5 is affected by gene polymorphisms, which present with individual differences (16). Thus, the present study aimed to investigate the gene expression ofCYP3A5, its enzyme activity and DNR concentrations, in order to provide experimental evidence for the use of DNR and reducingthe cardiac toxicity caused by DNR.

The present study identified three genotypes, CYP3A 5*1-"1, CYP3A5"1-"3 and CYP3A5*3-"3, in 36 children with ALL. PCR analysis was used to detect the mRNA expression of CYP3A5, which detected only a wt-mRNA (wt-CYP3A5) in the CYP3A5"1-"1 genotype, but identified two types of mRNA, wt and variant type (SV1-CYP3A5) in the CYP3A5 ${ }^{*}{ }^{*} 3$ and CYP3A5 3 -*3 genotypes. The metabolized clearance rate of MDZ ranged between 0.047 and 0.181 l-h-kg in the 36 children with leukemia. CYP3A enzyme activity combined with CYP3A5 genotype were analyzed, which revealed that CYP3A enzyme activity was higher in children with the CYP3A $5 * 1$ allele, compared with that in children with CYP3A $5 * 3$. Kuehl et al (17) indicated that the hydroxylation reaction rate to MDZ of individuals with a single CYP3A $5^{*} 1$ allele was at least twice as high as individuals with the CYP3A $5 * 3-{ }^{*} 3$

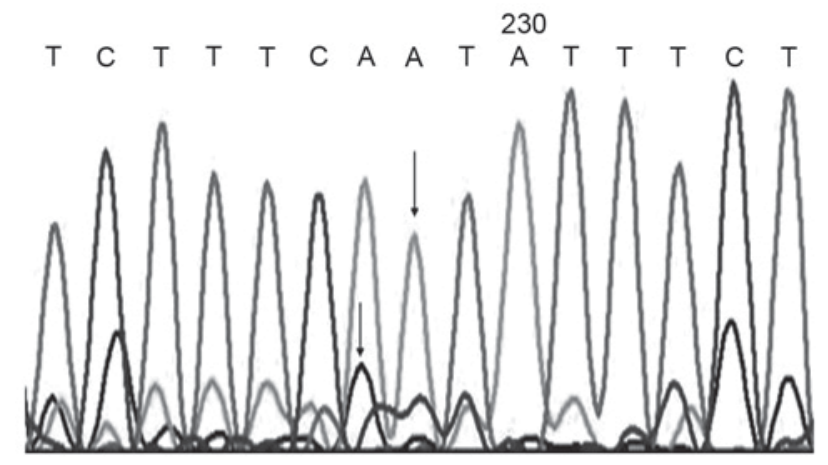

Figure 3. Cytochrome P450 family 3 subfamily A member $5 * 1-3$ gene sequencing diagram. Arrow shows two peaks of A and $\mathrm{G}$ in the site of 6,986 .

allele. Seng et al (18) found the CYP3A activity was higher in children with the CYP3A $5 * 1$ allele, compared with that in children with the CYP3A*3 allele, which was in accordance with the results of the present study.

In the present study, the monitoring of DNR blood concentrations was performed for $24 \mathrm{~h}$ in the 36 children with ALL on their initial visit to hospital using RHPLC. The results showed that the pharmacokinetics of DNR were significantly different in the 36 individuals, the difference in CL was over three times higher, and the AUC was four times higher in certain individuals. Kokenberg et al (19) investigated the pharmacokinetics of DNR in 21 patients with acute myeloid leukemia and found the AUC ranged between 0.06 and $0.37 \mathrm{nmol}-\mathrm{m}^{*} \mathrm{~h}$ between individuals. Bogason et al (20) also found the blood concentrations of DNR varied markedly in individuals $1 \mathrm{~h}$ following injection of DNR in 40 cases of acute myeloid leukemia, which ranged between 60 and 1,370 nM. The factors affecting the pharmacokinetics of DNR include heritage, age, pathology and environment, and hereditary factors determine the effect and toxicity of drugs (21). In the present study, correlation analysis between the mRNA expression of wt-CYP3A5 and metabolized clearance of DNR was performed in 36 children with ALL, and it was found that there was correlation between the two factors. The increase in the expression level of wt-mRNA led to an increase in metabolized drug clearance $(\mathrm{r}=0.769)$. Therefore, the detection of CYP3A5 genotype and phenotype are necessary for children with primary ALL prior to chemotherapy using DNR, in order to predict the metabolized clearance of DNR in the body, and to enhance the scientific value and rationality of DNR use.

The present study found that the AUC was markedly higher in four children with ALL with cardiac toxicity, compared with those without cardiac toxicity, which indicated that the DNR had high bioavailability, increasing the risk of cardiac toxicity. Therefore, the timely use of cardiac protectors is required, or a reduction in DNR dose is recommended. No reduction in the activity of CYP3A was observed in these four children, compared with children without cardiac toxicity. The detection of DNR pharmacokinetics required the collection of blood samples several times, which introduced difficulty in specimen collection. It is suggested that CYP3A activity is included in routine examinations prior to chemotherapy with DNR to predict the occurrence of cardiac toxicity following treatment with DNR. 
Table II. mRNA expression levels of CYP3A5 in patients with acute leukemia with different genotypes.

\begin{tabular}{lrcr}
\hline Genotype & $\mathrm{n}(\%)$ & Expression of wt-CYP3A5 & P-value \\
\hline CYP3A5*3-"3 & $22(61.1)$ & $0.73 \pm 1.29$ & $0.032^{\mathrm{a}}$ \\
CYP3A5*1-"3 & $10(27.8)$ & $6.12 \pm 2.23$ & $0.014^{\mathrm{b}}$ \\
CYP3A5 1-"1 & $4(11.1)$ & $21.36 \pm 12.57$ & $0.042^{\mathrm{c}}$ \\
\hline
\end{tabular}

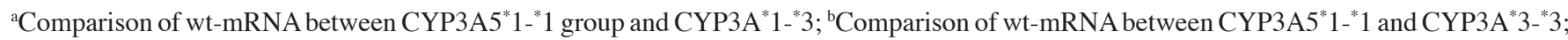
'Comparison of wt-mRNA between CYP3A5"1-"3 and CYP3A5*3-"3. CYP3A5, cytochrome P450 family 3 subfamily A member 5; wt, wild-type.

Table III. Pharmacokinetics of patients with different genotypes of cytochrome P450 family 3 subfamily A member 5 followinginjection of daunorubicin.

\begin{tabular}{lcrr}
\hline Parameter & $6986 \mathrm{GG}(\mathrm{n}=22)$ & 6986AG $(\mathrm{n}=10)$ & 6986AA $(\mathrm{n}=4)$ \\
\hline $\mathrm{t}_{1-2 \alpha}(\mathrm{h})$ & $0.59 \pm 0.14$ & $0.43 \pm 0.29$ & $0.38 \pm 0.11$ \\
$\mathrm{Vd}\left(1 \cdot \mathrm{m}^{-2}\right)$ & $319.27 \pm 120.11$ & $263.91 \pm 256.49$ & $157.04 \pm 92.38$ \\
$\mathrm{CL}\left(\mathrm{l} \cdot \mathrm{h}^{-2}\right)$ & $70.02 \pm 24.63$ & $79.81 \pm 29.14$ & $82.14 \pm 22.57$ \\
$\mathrm{AUC}_{0-24 \mathrm{~h}}(\mu \mathrm{g} \cdot 1-1 \cdot \mathrm{h})$ & $465.61 \pm 231.05^{\mathrm{a}}$ & $430.16 \pm 221.62$ & $305.16 \pm 241.62$ \\
$\mathrm{AUC}_{0-\infty}(\mu \mathrm{g} \cdot \mathrm{l}-1 \cdot \mathrm{h})$ & $586.10 \pm 382.15^{\mathrm{a}}$ & $503.10 \pm 282.24$ & $452.30 \pm 210.71$ \\
$\mathrm{~T}_{\max }(\mathrm{h})$ & $1.51 \pm 0.17$ & $1.37 \pm 0.25$ & $1.12 \pm 0.48$ \\
$\mathrm{C}_{\max }\left(\mu \mathrm{g} \cdot \mathrm{l}^{-1}\right)$ & $89.01 \pm 33.52$ & $74.66 \pm 33.52$ & $65.64 \pm 29.67$
\end{tabular}

Data are presented as the mean \pm standard deviation. ${ }^{\mathrm{a}} \mathrm{P}<0.03$, compared with the AA group. $\mathrm{t}_{1-2 \alpha}$, half-life; Vd, volume of distribution; CL, clearance; $A U C$, area under the curve; $\mathrm{T}_{\max }$, time to maximum concentration; $\mathrm{C}_{\max }$, maximum concentration.
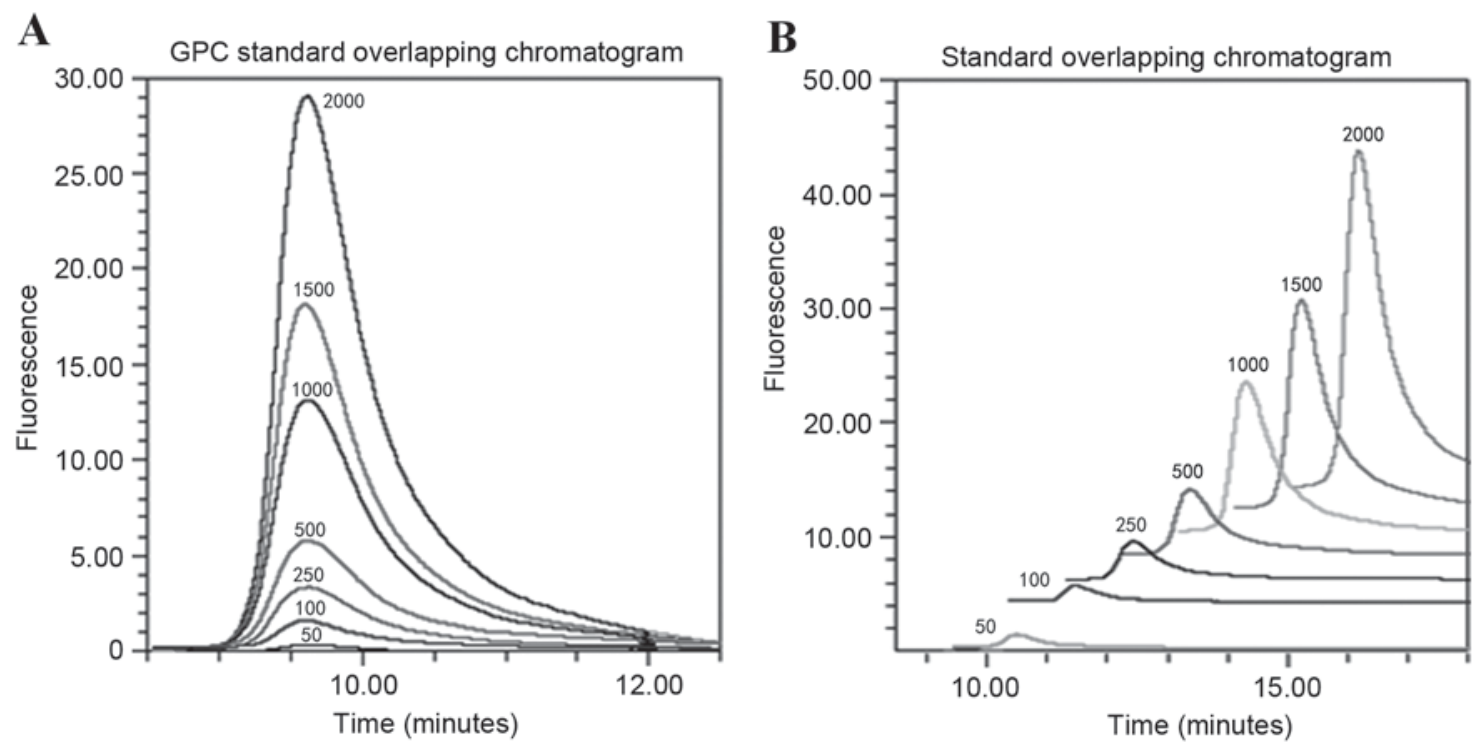

Figure 4. DNR chromatogram using high performance liquid chromatography. (A) The chromatogram of 50,100,250,500,1,000,1,500 and 2,000 ng.ml-1 DNR. (B) Partial enlarged view of retention time 10-15 min. DNR, daunorubicin.

In the process of treating leukemia, the detection of CYP3A5 gene polymorphisms and CYP3A enzyme activity, and monitoring the blood concentrations of anticancer drugs may provide objective indicators for individualized treatment and references for planning effective blood drug concentration rages to assist establishing individual treatment schemes.
In the present study, relatively few cases were enrolled and the observation duration was short. In addition, certain children may suffer from toxicity reactions as time progresses. Therefore, to confirm the association between efficacy and DNR pharmacokinetics, a larger sample size and longer observation period is required. 


\section{Acknowledgements}

The present study was supported by a grant from the Wenzhou City Science Plan (grant no. Y20160228).

\section{References}

1. Carvalho C, Santos RX, Cardoso S, Correia S, Oliveira PJ, Santos MS and Moreira PI: Doxorubicin: The good, the bad and the ugly effect. Curr Med Chem 16: 3267-3285, 2009.

2. Singal PK and Iliskovic N: Doxorubicin-induced cardiomyopathy. N Engl J Med 339: 900-905, 1998.

3. Wang Y, Zheng D, Wei M, Ma J, Yu Y, Chen R, Lacefield JC, $\mathrm{Xu} \mathrm{H}$ and Peng T: Over-expression of calpastatin aggravates cardiotoxicity induced by doxorubicin. Cardiovasc Res 98: 381-390, 2013.

4. Ohtake S, Miyawaki S, Fujita H, Kiyoi H, Shinagawa K, Usui N, Okumura H, Miyamura K, Nakaseko C, Miyazaki Y, et al: Randomized study of induction therapy comparing standard-dose idarubicin with high-dose daunorubicin in adult patients with previously untreated acute myeloid leukemia: The JALSG AML201 study. Blood 117: 2358-2365, 2011.

5. Goh BC, Lee SC, Wang LZ, Fan L, Guo JY, Lamba J, Schuetz E, Lim R, Lim HL, Ong AB and Lee HS: Explaining interindividual variability of docetaxel pharmacokinetics and pharmacodynamics in Asians through phenotyping and genotyping strategies. J Clin Oncol 20: 3683-3690, 2002.

6. Ro H, Min SI, Yang J, Moon KC, Kim YS, Kim SJ, Ahn C and Ha J: Impact of tacrolimus intraindividual variability and CYP3A5 genetic polymorphism on acute rejection in kidney transplantation. Ther Drug Monit 34: 680-685, 2012.

7. Kim KP, Ahn JH, Kim SB, Jung KH, Yoon DH, Lee JS and Ahn SH: Prospective evaluation of the drug-metabolizing enzyme polymorphisms and toxicity profile of docetaxel in Korean patients with operable lymph node-positive breast cancer receiving adjuvant chemotherapy. Cancer Chemother Pharmacol 69: 1221-1227, 2012.

8. Afsar NA, Ufer M, Haenisch S, Remmler C, Mateen A, Usman A, Ahmed KZ, Ahmad HR and Cascorbi I: Relationship of drug metabolizing enzyme genotype to plasma levels as well as myelotoxicity of cyclophosphamide in breast cancer patients. Eur J Clin Pharmacol 68: 389-395, 2012.

9. Lopes BA, Emerenciano M, Gonçalves BA, Vieira TM, Rossini A and Pombo-de-Oliveira MS: Polymorphisms in CYP1B1, CYP3A5, GSTT1, and SULT1A1 are associated with early age acute leukemia. PLoS One 10: e0127308, 2015.

10. Ma LM, Liu HC, Ruan LH and Feng YM: CYP3A5*3 genetic polymorphism is associated with childhood acute lymphoblastic leukemia risk: A meta-analysis. Biomed J 38: 428-432, 2015.
11. Liu X, Zou Y, Wang H, Chen X, Ruan M, Chen Y, Yang W, Guo Y, Liu T, Zhang L, et al: Treatment outcome of childhood standard-risk and median-risk acute lymphoblastic leukemia with CCLG-2008 protocol. Zhonghua Er Ke Za Zhi 52: 449-454, 2014 (In Chinese).

12. Florescu M, Cinteza M and Vinereanu D: Chemotherapy-induced cardiotoxicity. Maedica (Buchar) 8: 59-67, 2013.

13. Stankov K, Sabo A and Mikov M: Pharmacogenetic biomarkers as tools for pharmacoepidemiology of severe adverse drug reactions. Drug Dev Res 74: 1-14, 2013.

14. Francis J, Dubashi B, Sundaram R, Pradhan SC and Chandrasekaran A: A study to explore the correlation of ABCB1, ABCG2, OCT1 genetic polymorphisms and trough level concentration with imatinib mesylate-induced thrombocytopenia in chronic myeloid leukemia patients. Cancer Chemother Pharmacol 76: 1185-1189, 2015.

15. Hareedy MS, El Desoky ES, Woillard JB, Thabet RH, Ali AM, Marquet $\mathrm{P}$ and Picard N: Genetic variants in 6-mercaptopurine pathway as potential factors of hematological toxicity in acute lymphoblastic leukemia patients. Pharmacogenomics 16 : 1119-1134, 2015.

16. Fetterly GJ, Aras U,Lal D, Murphy M, Meholick PD and Wang ES: Development of a preclinical PK-PD model to assess antitumor response of a sequential aflibercept and doxorubicin-dosing strategy in acute myeloid leukemia. AAPS J 15: 662-673, 2013.

17. Kuehl P, Zhang J, Lin Y, Lamba J, Assem M, Schuetz J, Watkins PB, Daly A, Wrighton SA, Hall SD, et al: Sequence diversity in CYP3A promoters and characterization of the genetic basis of polymorphic CYP3A5 expression. Nat Genet 27: 383-391, 2001.

18. Seng KY, Hee KH, Soon GH, Sapari NS, Soong R, Goh BC and Lee LS: CYP3A 5*3 and bilirubin predict midazolam population pharmacokinetics in Asian cancer patients. J Clin Pharmacol 54: 215-224, 2014

19. Kokenberg E, Sonneveld P, Delwel R, Sizoo W, Hagenbeek A and Löwenberg B: In vivo uptake of daunorubicin by acute myeloid leukemia (AML) cells measured by flow cytometry. Leukemia 2: 511-517, 1988

20. Bogason A, Quartino AL, Lafolie P, Masquelier M, Karlsson MO, Paul C, Gruber A and Vitols S: Inverse relationship between leukaemic cell burden and plasma concentrations of daunorubicin in patients with acute myeloid leukaemia. Br J Clin Pharmacol 71: 514-521, 2011.

21. Chien JY and Ho RJ: Drug delivery trends in clinical trials and translational medicine: Evaluation of pharmacokinetic properties in special populations. J Pharm Sci 100: 53-58, 2011. 